\title{
Methodology and rationale for the measurement of harm with trigger tools
}

\author{
R K Resar, J D Rozich, D Classen
}

Qual Saf Health Care 2003;12(Suppl II):ii39-ii45

The growing recognition of harm as an unwelcome and frequently unrecognized byproduct of health care has initiated focused efforts to create highly reliable organizations for safe healthcare delivery. While debate continues over the exact magnitude of harm, there is a general acceptance of the need to improve our ability to deliver care in a safer manner. A major barrier to progress in safety has been the ability to effectively measure harm consistently and thus develop effective and targeted strategies to prevent its occurrence. This has resulted in a shiff from initiatives focused exclusively on analysis of errors to those targeting events linked to harm. There is a growing recognition of a distinction between errors and adverse events as they often represent unique concepts fostering different strategies for improvement of safety. Conventional approaches to identifying and quantifying harm such as individual chart audits, incident reports, or voluntary administrative reporting have often been less successful in improving the detection of adverse events. As a result, a new method of measuring harm -the trigger tool-has been developed. It is easily customized and can be readily taught, enabling consistent and accurate measurement of harm. The history, application, and impact of the trigger tool concept in identifying and quantifying harm are discussed.

See end of article for authors' affiliations

Correspondence to: Dr J D Rozich, Chief of Cardiology, Luther Midelfort, 1400 Bellinger Street, Eau Claire, Wl 54702, USA; rozich.john@mayo.edu
$\mathrm{T}$ here is widespread recognition that health care is in need of fundamental change to improve the safety of care for patients. Two recent reports by the Institute of Medicine have highlighted concerns that perhaps thousands of deaths each year are related to errors in health care. ${ }^{12}$ Efforts to identify and understand these errors have been made and solutions based on practical and effective responses have been sought. Unfortunately, historical evidence and practical experience have led to an evolving realization that efforts directed solely at identifying and quantifying errors have not optimized the safety of care. ${ }^{3-8}$ The explanation for this is complex and deserves clarification because an understanding of this perceptual paradox has led safety experts to consider very different approaches in their attempts to prevent patient harm. A conceptual model has been advanced suggesting that the metrics of latent errors, active errors, and adverse events are intrinsically different. ${ }^{8}$ Each requires a contextual understanding of its specific potential contribution to safety. Importantly, the largest source of errors involves medications, and the significant majority of these do not result in harm to patients. ${ }^{369}$ They are either insignificant and include mistakes in dosages or times of administration that are clinically benign or they are errors that are discovered before execution. Thus, simply focusing upon errors may not adequately link a mistake to human harm. ${ }^{3-6}$

Any new approach to the analysis of harm must therefore account for actual patient injury and clarify the contributing source. ${ }^{4}$ In addition, safety must be an inherent property of the system or organization to result in decreased harm..$^{4-6} 910$ Thus, efforts to improve safety should include the ability to know the underlying cause and nature of events that injure patients. Simply detecting and quantifying errors may not establish the root cause of repetitive mishaps that instead reflect a deficiency in the system of care. In this paper we describe a new practical methodology to reliably identify, quantify, and track events related directly to patient harm. This methodology uses "triggers" to identify adverse events in the medical record that are ultimately linked to patient harm, enabling both active and latent errors to be more directly related to harm. ${ }^{35611-15}$

\section{DISTINCTION BETWEEN ERRORS AND ADVERSE EVENTS}

The overall goal of improved safety in health care is to reduce patient injury or harm. ${ }^{4-6}{ }^{16-18}$ This underscores the importance of distinguishing between errors and adverse events. Medical errors are failures in the process of care and, while they have the potential to be harmful, numerous reports have shown that they are often not linked to the injury of patients. ${ }^{3-6}$ Instead, they are often "caught" by the system before they can lead to injury. ${ }^{4-6}$ It is noteworthy that, even when errors reach the patient, they are most often minor and, in most instances, result in no significant damage. Examples are common in medicine-for instance, a drug being given an hour later than prescribed or even the wrong drug being given to the patient, such as diphenhydramine being given incorrectly. This is an error but, in the overwhelming number of instances, administering a small dose of diphenhydramine (even to the wrong patient) will not result in harm.

In direct contrast, adverse events are directly linked to actual harm resulting from medical care. ${ }^{4-6}$ Adverse events are thus any injury caused 
by medical management and are independent of the patient's disease. An adverse event may also occur despite the correct care being given under correct circumstances but ultimately associated with a poor outcome. Thus, performing an invasive diagnostic test for the correct medical indication and even correctly establishing the diagnosis may still be associated with an adverse event if there is an unforeseen complication such as infection or bleeding resulting from this invasive testing. No error has occurred in this example, yet harm has resulted.

In the Idealized Design of the Medication System, the Institute for Healthcare Improvement has championed a conceptual approach to enhance safety, emphasizing the distinction between errors and harm..$^{3-5}$ Currently, no single comprehensive or easily understood terminology exists..$^{3-6} 18$ The definition of a medical error includes the failure of a planned action to be completed as intended or the use of a wrong plan to achieve an aim. The definition of an adverse event is any injury (not just that associated with medication or drug use) caused by medical management rather than the underlying condition of the patient..$^{5-711} 12$ This is a more general definition than that provided by the World Health Organization, which describes an adverse drug event (ADE) as "a response to a drug, treatment or intervention which is noxious and unintended and which occurs at doses or methodology normally used in man for prophylaxis, diagnosis, or therapy of disease, or the modification of physiological function". ${ }^{19}$ However, both definitions are conceptually linked directly to harm suffered by the patient. The more general definition thus encompasses all events associated with patient harm, not just those linked to medication related injury. While medication associated events (ADEs) remain the most common source of patient injury because drugs are the single most common intervention, accurate indices of harm must be more comprehensive. Harm must also be examined from the patient's perspective. The patient frequently has no appreciation as to whether an error was associated with the outcome, but he or she can readily decide if harm occurred in relation to a mishap. Safety then becomes a definition of "no harm". Harm for the purposes of this paper is conceptually limited to physical injury. Our intent is not to exclude mental or emotional harm from the overall equation, but rather to start from a definable base that most organizations can begin to work with effectively.

There are several distinct advantages in attempting to quantify harm instead of errors. ${ }^{4-6}$ As noted above, errors are process focused and often end up exclusively examining an individual's role in a real or potential mishap. ${ }^{4} 1112$ Alternatively, a focus on harm can target the system rather than the individual, and explores methodology to improve or enhance clinical outcomes. This has the practical effect of reducing punitive concerns associated with the methodology and fosters greater compliance. ${ }^{2021}$ Since the process examines all unintended results, the definition of harm is more comprehensive and grounded in what the patient experiences. The finding of adverse events can thus contribute to a continual process of improvement without fears of punitive action by those involved. ${ }^{56}$ The concept also accepts a responsibility for the "unavoidable or unforeseen" adverse events. These "unavoidable" adverse events—such as antibiotic resistance in a nosocomial infection-become amenable to changes in the system of care being delivered..$^{56}$

If harm is the variable examined through its impact on clinical outcomes, effort is redirected to correcting the operational system rather than assigning blame to individuals. ${ }^{20}$ This knee jerk response ignores the prescient view that "a system is designed to deliver the exact results it delivers".$^{20}$ Focusing on harm allows analysis of unintended results despite operational compliance and encourages learning from events to continually improve the process. Quantifying harm is also patient centred, with current evidence indicating that consumers are demanding a safer medical environment. Accountability is also established as agreement is reached regarding the acceptance that harm, not individuals to be punished, is the relevant variable.

As the basis for the distinction between errors and adverse events has become better understood, the need for a reliable metric of harm has become apparent. Because one cannot improve upon that which cannot be identified or measured, there has been considerable enthusiasm for a methodology which uses "triggers" to "unsurface" harm. . $^{311-13}$

\section{METHODOLOGIES FOR MEASUREMENT OF HARM}

Conventional attempts to quantify harm have included incident reports, retrospective or concurrent chart reviews, and observational data. Each of these methodologies has been used to assess the origins of safety in health care but all have suffered from various shortcomings. Incident reports have attempted to identify specific events that endanger or could lead to injury of patients, but systematic evaluation of their efficacy reveals near universal underutilization. ${ }^{3-6}$ This lack of use is thought to be related to pressures of the work place that demand that the work gets done and the incident report competes for time in an environment where nurses and staff are already stretched beyond their capacities. A more powerful disincentive is the tacit belief that these are associated with punitive repercussions. Numerous survey responses show that nurses and hospital employees candidly emphasize that incident reports may negatively impact on their career or that of their colleagues.

Retrospective or concurrent chart reviews are time consuming and may be inaccurate. The variability between results generated by reviewers is a reflection of the need for a basic medical professional understanding of the complex data found in the medical record. Reviews also suffer from a lack of consistency as to what constitutes an event and agreement on how to classify or prioritize harm. A component of this inconsistency is the inability to extract meaningful data from the medical record related to poor or incomplete entries or confusing or conflicting data. Chart reviews are also resource intensive in both time and money. Observational data have also been ineffective in identifying or quantifying harm. As previously noted, there has been little historical consensus on what constitutes harm, obstructing attempts to recognize or quantify it. The conceptual distinctions between errors and adverse events have also hampered the effectiveness of conventional retrospective or concurrent chart reviews.

\section{TRIGGER TOOL METHODOLOGY}

The concept of a "trigger" or a sentinel word to identify adverse events in the medical record was introduced by Jick in 1974..$^{22}$ The approach was refined and automated using electronic triggers with an integrated hospital information system to isolate adverse events. ${ }^{13}{ }^{14}$ In this system, specific events-including the ordering of certain drugs, orders for antidotes, certain abnormal laboratory values, and abrupt stop orders-serve as sentinels or triggers to initiate a more detailed concurrent chart audit. Each time a trigger event was found in the pharmacy or physician order sheet of the medical record it was counted and referenced. Every day a report of the patients identified with possible ADEs was provided to a pharmacist for further in depth concurrent review. This purely electronic initial screening strategy coupled with real time evaluation accomplishes a rapid review of a patient's current record for the occurrence of ADEs. 
Various applications of this concept have been used by Hurwitz, ${ }^{23}$ Jick, $^{22}$ and McMullin ${ }^{24}$ and others to probe the medical record. ${ }^{12-1425} \mathrm{~A}$ more generalizable form of trigger methodology was recently adapted by the Idealized Design of the Medication System team for the measurement of ADEs, ${ }^{3}$ and the tool has now been used by more than 200 organizations. The goal was to combine Classen's conceptual strategy using triggers with methodology that did not require computerized technology and thus the requisite infrastructure expense. Recent publications have reported that the trigger methodology produces consistent, reliable, and relevant data at low cost. ${ }^{3}$ The ability to measure harm using a low technological approach at reduced cost enables expanded application of the tool. A secondary goal of introducing this more generalizable metric was to initiate the process of adopting universally accepted terminology and standards for reporting of trigger derived data.

The methodology for all trigger tools is based on a chart selection process. For ADEs in a general hospital setting a random chart selection is employed. ${ }^{12-14} 25$ These charts are reviewed using a predetermined list of specified triggers that are associated with ADEs. For example, to identify a spectrum of ADEs, triggers would consist of "use of Narcan", "rising serum creatinine", or perhaps "use of diphenhydramine". But not all positive triggers necessarily identify an ADE. This fact is more easily appreciated if one considers the use of diphenhydramine as a sedative or as an antihistamine for a drug reaction. The use of diphenhydramine as a sedative is not a trigger associated with an ADE, but use of this same agent in response to a drug reaction or urticaria represents a valid trigger for an ADE. A review of the relevant section of the medical record will therefore clarify the use of the agent and the context of use for any of the specified triggers.

Chart reviews and observational data have traditionally examined errors with the assumption that preventing errors will also reduce adverse events. ${ }^{3-7} 9$ Even as this conventional approach began to recognize that errors were infrequently associated with real patient harm, the belief persisted that aggressive prevention would eventually have an impact on events associated with patient injury. The trigger methodology is predicated on the hypothesis that surveillance of events that are tightly linked to harm will enable a more powerful strategy to reduce injury. ${ }^{3-5}$ This approach emphasizes identifying and documenting adverse events that have been clearly shown to have a higher net yield-for it is harm that is the focus, not benign errors or innocent mistakes. The long range strategy is that, by identifying a very high percentage of relevant safety concerns, interventions may be developed to successfully combat systems that result in suboptimal patient care or outcomes.

Four separate functional trigger tools have been developed and tested (table 1). The first is the general ADE trigger tool developed by the IHI and Premier in the Idealized Design of the Medication System. ${ }^{3}$ The ADE trigger tool has been used by over 200 organizations associated with either IHI, VHA, or Premier sponsored safety collaboratives. ${ }^{3}$ The basic tool has 24 triggers and about 20 minutes is required to review an average inpatient chart (Appendix 1). The tool has been used to examine the frequency and types of ADEs found during the management of hospital inpatients. It has also been used to follow the "harm burden" (a metric of total identifiable harm) of an organization longitudinally through time. The benefit of being able to quantify harm and the change in these rates as corrective initiatives are exciting new developments. The baseline for US hospitals appears to be about 2.64 ADEs per 1000 doses of medication or $24 \%$ of all admissions. ${ }^{3}$ One lesson learned from the large group of organizations participating in the IHI/Premier collaborative is the relative ease with which the tool can be taught and used. It can be taught to qualified professionals in approximately 30 minutes, $^{3}$ and it is also readily adaptable for use in the electronic medical record that is similar to Classen's original report. ${ }^{13}$

The second trigger tool that has been tested is the ICU adverse event tool (Appendix 2). This was initially tested by a VHA/IHI intensive care collaborative and has now been incorporated into an IMPACT domain, a larger IHI program. ${ }^{27}$ This tool also uses 24 triggers but looks at all adverse events rather than just ADEs. The methodology again uses small samples of random chart reviews, about 10 charts being audited every 2 weeks. These audits are continued over time with a reviewer examining only the time spent in the ICU for each chart. In this manner the redesign teams reviewed 1300 charts and detected an adverse event rate per day of $0.164 .^{3}$ Of the total admissions to the ICU, 55\% experienced at least one adverse event and $28 \%$ experienced more than one. Although drug related adverse events represented $18 \%$ of the total, the most common triggers were intubation or reintubation, oversedation, and decreasing hemoglobin levels of $>4 \mathrm{~g} / \mathrm{dl}$. It must be reiterated that, once a trigger such as intubation is found, auditing the chart determines whether a true adverse event occurred. This approach obviates concern among practising clinicians that "intubation in the ICU" is often an anticipated outcome and therefore cannot be classified as an adverse event. Given that intubation is more frequent in the ICU, it was nevertheless noted to be an adverse event during chart review either because it was not anticipated or secondary to avoidable aspects of the care management process.

The third use of the trigger tool is as a process specific tool-for example, the "warfarin trigger" column in table $1 .{ }^{26}$ The tool is particularly helpful for examining adverse events in greater detail for more intensive evaluation of a given process. Too frequently the measurements needed and ultimately used to validate the need for operational change become a process measure rather than an outcome measure. $^{328}$ In other words, the measurement process itself takes on a "life of its own" and submerges its value as a metric of relevant clinical outcomes. This is best exemplified in the examination of warfarin use an anticoagulant. Anticoagulation is the most common drug related to adverse medication events using the standard ADE trigger tool. ${ }^{3}$ Organizations have used different "triggers" including abnormal international normalized ratios (INRs), clinical evidence of bleeding, or progressive development of anemia to identify outcomes related directly to harm. Thus, the trigger enables extraction of data to analyze the conditions that describe the process involved in using the anticoagulant warfarin. This approach obviates efforts that can proceed to the process of measurement itself becoming the actual goal or end result. Basing decisions on outcomes associated with the safety of care enables clinicians to measure the harm burden associated with warfarin use and to quantify the effectiveness of introducing changes to its use such as protocols for standardizing the dosage. ${ }^{3-5}$

Finally, triggers are also being customized for use in the outpatient or ambulatory care setting. In this environment, triggers have been modified by using them within the concept of "life events". As an example (see table 1, third column), patients will identify with certain health care related occurrences such as emergency department visits, a new diagnosis of cancer, or an abnormal laboratory or test result. These are the "life events" and they can be used along with associated triggers to probe further into why or how specified events occurred and how they were processed by the healthcare system. For example, if the patient is visiting the emergency department (the life event) secondary to seeking treatment for an adverse event such as a reaction to a 


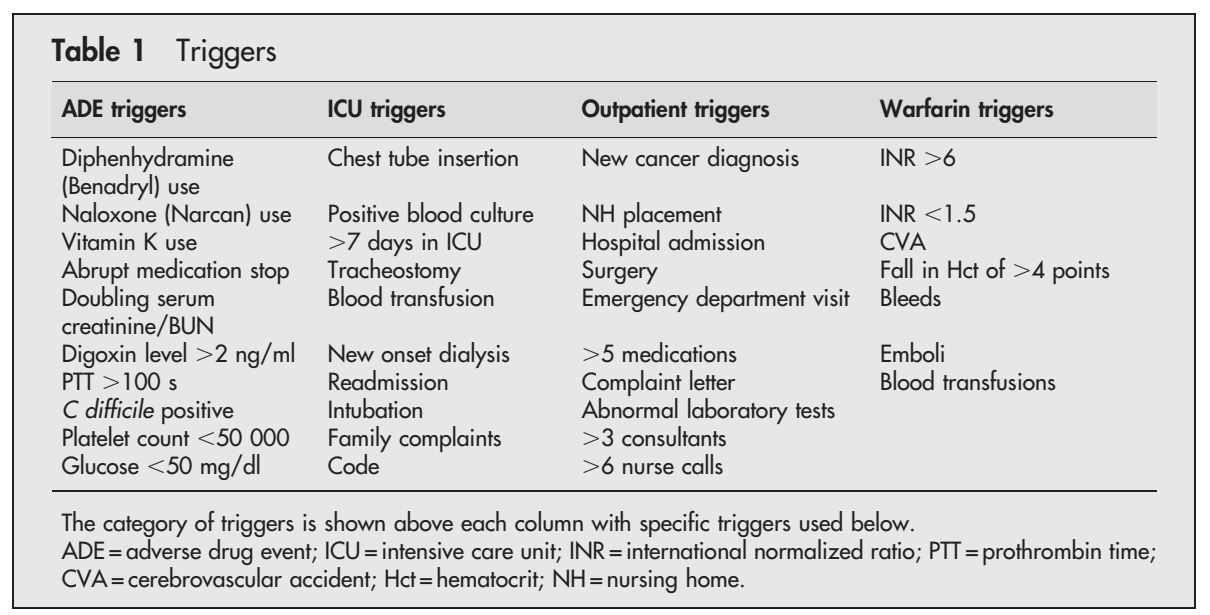

medication, this information can now be identified and quantified. Reviewing this record will determine if specified triggers are present and if the visit was in response to system related harm. In one organization 200 care years were reviewed in less than 4 hours ( $\mathrm{R} K$ Roger, personal communication, 2001). For those patients over the age of 60 who had at least two visits to the outpatient department, an adverse event rate of $17 \%$ per year was reported. When applied to life events, the trigger tool can be used to examine harm in the ambulatory care setting in a manner not previously feasible. The value of the trigger tool is the ability to customize it to the specific environment, to derive useful data, and to follow the impact of any corrective interventions (fig 1).

\section{PRIMARY OBJECTIVE OF TRIGGER METHODOLOGY}

As the experience with trigger tool methodology has grown, several fundamental characteristics have come to light. The trigger tool process is a strategy for detecting adverse events or outcomes because they are tightly linked to patient harm..$^{3-5}$ The focus is detection, quantification, and tracking of adverse outcomes, which enables accurate examination of harm in different environments in health care. Ultimately, the trigger tool provides a process for direct intervention by

Fill out the following information below for each DISTINCT adverse event with a harm category of E and above:

Harm category*

$(\mathrm{E} / \mathrm{F} / \mathrm{G} / \mathrm{H} / \mathrm{I})$

Event \#1

Event \#2.

Event \#3.

Event \#4

Event \#5

Event \#6

Event \#7

*Harm categories

Category E: contributed to temporary harm to the patient and required intervention

Category F: contributed to temporary harm to the patient and required initial/prolonged hospitalization

Category G: contributed to permanent patient harm

Category $\mathrm{H}$ : required intervention to sustain life

Category I: contributed to the patient's death

\author{
** Medication code \\ 0-Not applicable \\ 1-Antibiotics \\ 2-Anticoagulants and antiplatelets \\ 3-Antipsychotics \\ 4-Electrolytes \\ 5-Insulin \\ 6-Narcotics \\ 7-Sedatives
}

***Was the adverse event documented as an "E" code in the ICD9 section of the UB92 form?

Yes-only pertains to documentation of the following "E" codes: Accidental poisoning (E850-E869); Therapeutic use (E930-E949); Suicide attempt (E950-E952); Assault (E961-E962); Undetermined (E980-E982)

Figure 1 Identification of harm category based on the adverse event. This allows quantification of the direct linkage of the adverse event to a clinical outcome. 


\section{Pointers for future research}

- Universal use of trigger tool methodology to identify and quantify harm.

- Greater use of automated methodology.

- Expanded use in all clinical settings.

organizations to impact on the system of care. In other words, the tool builds a measurement infrastructure wherein data can be used to create safer medical environments. Every organization is able to initiate the trigger tool methodology economically by beginning with the manual approach. Some will move quickly to automate the process. The technique is flexible and can be customized to specific organizations, locations within these entities, and may be refined for targeted areas within acute care facilities such as intensive care units or diagnostic heart catheterization laboratories. The technique offers the ability to quantify accurately with relatively small samples and to follow changes longitudinally over time. In addition to computerized or electronic scanning approaches, a manual approach with trained auditors can be used which requires little capital investment. ${ }^{311-14} \mathrm{~A}$ benefit of using the manual trigger tool methodology is that it brings the participant to the "front line" of data collection. This may confer an advantage by coupling reviewers directly to system users and thus making proposed improvements relevant.

Focused activity directed at reducing harm within the healthcare system will undoubtedly require many different innovative strategies. Significant progress has already been made in the administration of pharmacological therapeutics. Using the trigger tool methodology, several organizations have reduced their ADEs by more than $50 \%$ in 6 months. ${ }^{28}$ ADEs continue to be the single largest source of recurrent incidents placing patients at risk for harm. This is not surprising given that pharmacological intervention is so widespread, individuals are often receiving several different drugs, and the system in place to facilitate therapeutics is varied and often complex.

Growing recognition of harm in medicine has focused efforts to quantify and track responses that are then introduced to improve conditions. Adverse events continually harm patients but frequently are difficult to uncover and quantify using conventional methodologies. In fact, traditional methods to identify events (including incident reports and conventional chart reviews) have failed to provide a reliable metric for assessing harm. The evolution of the trigger tool into a more general method for investigating practice patterns provides a powerful new conceptual framework to understand, quantify, and track harmful events. Perhaps the most exciting future use of the trigger tool is its ability to follow corrective initiatives over intervals of time and to assess their impact. It should be appreciated that the trigger tool is not in itself an "improvement methodology", but it enables data acquisition and subsequent analysis of areas where harm is occurring and where resources need to be allocated to correct the problem. Quantifying harm for the sake of measurement "activity" is non-productive. Instead, the trigger tool should be used to produce a safer medical environment

\section{CONCLUSIONS}

Improved safety for patients is a universal priority in health care. However, efforts to impact meaningfully on safety and to reduce harm have been slowed by methodologies that fail to identify and quantify relevant clinical mishaps accurately. Simply identifying errors has been less effective in reducing harm, perhaps because of their poor linkage to the actual clinical injury. The trigger tool is a relatively simple method which permits consistently accurate identification and measurement of a broad range of adverse events that are directly linked to clinical harm. The methodology is flexible, and is able to function in a variety of clinical environments from outpatient ambulatory care to the intensive care unit. It can be used without significant set up costs or infrastructure using the "low tech" manual approach, yet it can also be incorporated into sophisticated computer scanning software. Ultimately, the trigger tool should provide an additional instrument for improving safety by allowing reliable measurements of patient injury.

\section{APPENDIX 1: RATIONALE FOR ICU TRIGGERS}

Appendix 1 lists the specific triggers and the rationale for their use. Other site specific triggers can be used and modification of existing triggers is acceptable. A basis for their modification and use is predicated on identifying adverse events to improve safety.

\begin{tabular}{|c|c|}
\hline Triggers & Rationale for use \\
\hline Positive blood culture & $\begin{array}{l}\text { Blood stream infections are frequently iatrogenic. } \\
\text { Commonly associated with poor outcomes in the } \\
\text { ICU }\end{array}$ \\
\hline $\begin{array}{l}\text { Abrupt fall in Hct or } \\
\mathrm{Hg} \text { of } \geqslant 4 \text { points }\end{array}$ & $\begin{array}{l}\text { Good trigger for bleeds related to surgery or } \\
\text { procedure complications. Also picks up } \\
\text { anticoagulant problems. Deals with bleeds of all } \\
\text { kinds }\end{array}$ \\
\hline $\begin{array}{l}\text { Clostridium difficile } \\
\text { positive }\end{array}$ & Related to antibiotic usage \\
\hline PTT and INR & $\begin{array}{l}\text { Anticoagulant related problems. Very common } \\
\text { in the ADE tool due to high risk nature of } \\
\text { anticoagulants }\end{array}$ \\
\hline Glucose $<50 \mathrm{mg} / \mathrm{dl}$ & $\begin{array}{l}\text { Insulin is a known high risk drug which accounts } \\
\text { for a number of ADEs }\end{array}$ \\
\hline $\begin{array}{l}\text { Rising BUN and } \\
\text { serum creatinine }\end{array}$ & $\begin{array}{l}\text { Ties renal dysfunction related to drugs and other } \\
\text { therapies }\end{array}$ \\
\hline $\begin{array}{l}\text { Radiological tests for } \\
\text { clot or emboli }\end{array}$ & $\begin{array}{l}\text { Clot formation and emboli occur in units due to } \\
\text { immobility }\end{array}$ \\
\hline $\begin{array}{l}\text { Sodium polystyrene } \\
\text { (Kayexalate) }\end{array}$ & $\begin{array}{l}\text { Will pick up potassium toxicity and worsening } \\
\text { renal function }\end{array}$ \\
\hline $\begin{array}{l}\text { Diphenhydramine } \\
\text { (Benadryl) }\end{array}$ & Frequently the only hint of an allergic reaction \\
\hline Vitamin K & $\begin{array}{l}\text { Coumadin reversal frequently the result of over } \\
\text { anticoagulation and bleeding complications }\end{array}$ \\
\hline $\begin{array}{l}\text { Flumazenil } \\
\text { (Romazicon) }\end{array}$ & $\begin{array}{l}\text { Sedative reversal agent. Related to episodes of } \\
\text { lethargy and hypotension }\end{array}$ \\
\hline Naloxone (Narcon) & Pain medication related complications \\
\hline $\begin{array}{l}\text { Antidiarrheals/ } \\
\text { laxatives }\end{array}$ & $\begin{array}{l}\text { May seem elemental but give clues to } C \text { difficile } \\
\text { infections and constipation. Constipation in the } \\
\text { unit can be a very serious problem. }\end{array}$ \\
\hline Antiemetics & May specify toxic level of drug or ADE \\
\hline Code & $\begin{array}{l}\text { All codes need to be carefully reviewed as the } \\
\text { end event of a flawed care process. Not all codes } \\
\text { are adverse events }\end{array}$ \\
\hline $\begin{array}{l}\text { Pneumonia onset in } \\
\text { unit }\end{array}$ & $\begin{array}{l}\text { By definition, all nosocomial pneumonias will be } \\
\text { adverse events }\end{array}$ \\
\hline Readmission to ICU & $\begin{array}{l}\text { Need to determine if the transfer out was too } \\
\text { premature and if the event had origins in the ICU }\end{array}$ \\
\hline New onset dialysis & $\begin{array}{l}\text { Frequently an end event of major intensive care } \\
\text { problems }\end{array}$ \\
\hline $\begin{array}{l}\text { Procedures while in } \\
\text { the unit }\end{array}$ & Evaluate for causation \\
\hline Intubation/reintubation & These processes are frequently related to events \\
\hline $\begin{array}{l}\text { Abrupt medication } \\
\text { stop }\end{array}$ & $\begin{array}{l}\text { When high risk medications are discontinued } \\
\text { abruptly these frequently indicate complications }\end{array}$ \\
\hline $\begin{array}{l}\text { Oversedation/ } \\
\text { lethargy/hypotension }\end{array}$ & $\begin{array}{l}\text { A nebulous category, but one of the most } \\
\text { frequently associated with events in the } \\
\text { medication triggers }\end{array}$ \\
\hline Other & $\begin{array}{l}\text { When the chart is reviewed an event is frequently } \\
\text { uncovered that fits none of the triggers. This is the } \\
\text { trigger that would be identified }\end{array}$ \\
\hline $\mathrm{Hc}$ & $\begin{array}{l}\text { nemoglobin; } \mathrm{PTT}=\text { prothrombin time; } \\
\text { malized ratio. }\end{array}$ \\
\hline
\end{tabular}


APPENDIX 2: ICU ADVERSE EVENT TRIGGERS

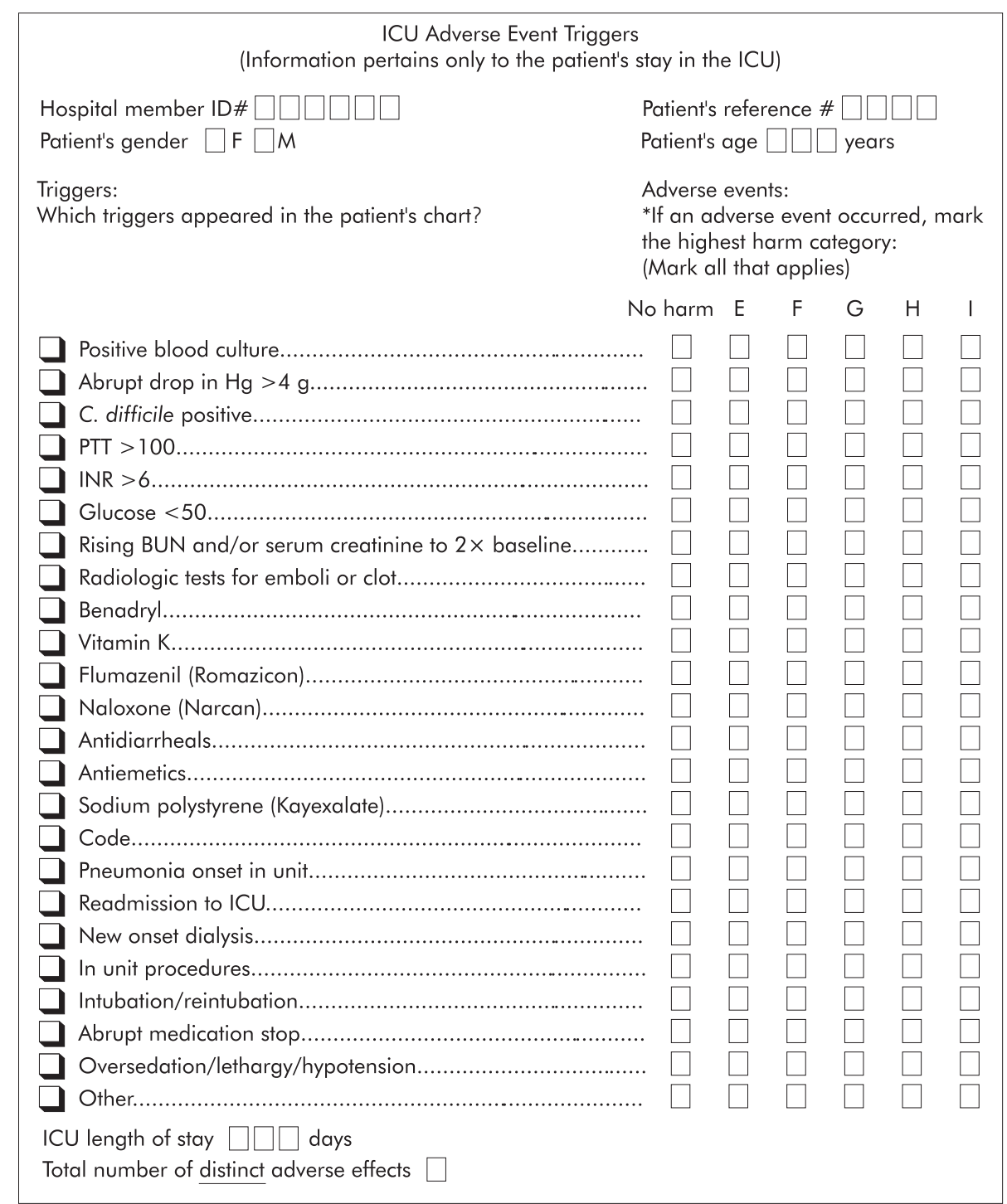

Authors' affiliations

R K Resar, J D Rozich, Luther Midelfort, 1400 Bellinger Street, Eau Claire, WI 54702, USA

D Classen, University of Utah, USA

\section{Key messages}

- Trigger tool methodology focuses on detecting, quantifying, and tracking adverse outcomes.

- Trigger tool methodology is related to actual clinical injury.

- It is flexible, and can be used in all clinical environments and to detect multiple types of adverse events.

- It is inexpensive and can be introduced without significant technology.

- It is consistent and accurate in measuring adverse events.

\section{REFERENCES}

1 Kohn L, Corrigan J, Donaldson M, eds. To err is human: building a safer health system. Washington, DC: National Academy Press, 1999.

2 Richardson WC, Briere R, eds. Crossing the quality chasm: a new health system for the 21st century. Washington, DC: National Academy Press, 2001.

3 Rozich JD, Haraden CR, Resar RK. The adverse drug event trigger tool: a practical methodology for measuring medication-related harm. Qual Saf Health Care 2003;12:194-200.

4 Layde PM, Maas LA, Teret SP, et al. Patient safety efforts should focus on medical injuries. JAMA 2002;287:1993-7.

5 Kilbridge $P$, Classen D. Surveillance for adverse drug events: history, methods and current issues. VHA Research Series 2002;3:1-48.

6 Kilbridge $P$, Classen DC. A process model of inpatient medication management and information technology to improve patient safety. VHA Research Series 2001;1:1-96.

7 Classen DC. Adverse drug events and medication errors: the scientific perspective. In: Scheffler AL, Zipperer LA, eds. Proceedings of Enhancing Patient Safety and Reducing Errors in Health Care. Chicago, IL: National Patient Safety Foundation, 1999.

8 Cullen DJ, Bates DW, Small SD, et al. The incident reporting system does not detect adverse drug events: a problem for quality improvement. Jt Comm J Qual Improv 1995;21:541-8.

9 Thomas EJ, Petersen LA. Measuring errors and adverse events in health care. J Gen Intern Med 2003;18:61-7.

10 Leape LL, Berwick DM, Bates DW. What practices will most improve safety? JAMA 2002;288:501-7.

11 Classen DC, Pestotnik SL, Evans RS, et al. Adverse drug events in hospitalized patients. Excessive length of stay, extra costs, and attributable mortality. JAMA 1997:277:301-6. 
12 Classen DC, Metzger J. Improving medication safety: the measurement conundrum and where to start. First Consulting Group 2002.

13 Classen DC, Pestotnik SL, Evans RS, et al. Description of a computerized adverse drug event monitor using a hospital information system. Hosp Pharmacy 1992;27:774-83.

14 Evans RS, Pestotnik SL, Classen DC, et al. A computer-assisted management program for antibiotics and other antiinfective agents. N Engl J Med 1998;338:232-8

15 Bates DW, Boyle DL, Vander Vliet VM, et al. Relationship between medication errors and adverse drug events. J Gen Intern Med 1995; 10:199-205.

16 Brennan TA, Leape LL, Laird N, et al. Incidence of adverse events and negligence in hospitalized patients. N Engl J Med 1991;324:370-6.

17 Leape LL, Brennan TA, Laird N, et al. The nature of adverse events in hospitalized patients. Results of the Harvard Medical Practice Study II. N Engl J Med 1991;324:377-84.

18 Bates DW, Cullen DJ, Laird NM, et al. Incidence of adverse drug events and potential adverse drug events: implications for prevention. JAMA 1995;274:29-34.

19 ICH Steering Committee. ICH harmonized tripartite guideline. Clinical safety data management: definitions and standards for expedited reporting. Workbook for investigators. Section 1. 27 October 1994:3.

20 Berwick DM. Not again! Preventing errors lies in redesign-not exhortation. BMJ 2001;322:247-8.

21 Reason J. Human error: models and management. BMJ 2000;320:768-70.

22 Jick H. Drugs - remarkably toxic. N Engl J Med 1974;291:824-8.

23 Hurwitz N, Wade OL. Intensive hospital monitoring of adverse reactions to drugs. BMJ 1969;1:531-6.

24 McMullin ST, Reichley RM, Kahn MG, et al. Automated system for identifying potential dosage problems at a large university hospital. Am J Health Syst Pharm 1997; 54:545-9

25 Payne TH, Savarino J, Marshall R, et al. Use of a clinical event monitor to prevent and detect medication errors. Proceedings AMIA Symp 2000:640-4. 26 World Health Organization. Presentation at Critical Care Summit. Chicago: VHA/IHI Intensive Care Collaborative, 21 May 2002.

27 Resar RK. Quantum leaps in patient safety. Institute for Healthcare Improvement, Learning Session \#1, October 2001.

28 Rozich JD, Resar RK. Medication safety: one organization's approach to the challenge. J Clin Outcomes Manage 2001;8:27-34 